\title{
Clonal Analysis of Staphylococcus hominis Strains Isolated from Hospitalized Patients
}

\author{
EWA SZCZUKA*, KRZYSZTOF TRAWCZYŃSKI and ADAM KAZNOWSKI \\ Department of Microbiology, Institute of Experimental Biology, Faculty of Biology \\ Adam Mickiewicz University, Poznań, Poland
}

Submitted 10 January 2013, revised 30 July 2014, accepted 30 July 2014

\begin{abstract}
Staphylococcus hominis is a part of normal skin flora, but it is also a cause of nosocomial infections. The aim of this study was to investigate the genetic relatedness of 62 strains of $S$. hominis obtained from hospitalised patients during an 11-year period. For the discrimination of these clinical strains we used repetitive sequence-based PCR method (BOX-PCR) and multiple-locus variable-number tandem repeat analysis (MLVA). BOX-PCR analysis revealed a large genetic diversity among clinical strains and we did not find a predominant clone with the ability to persist in a hospital environment. MLVA is not as discriminatory as BOX fingerprinting and would not be a useful method for epidemiological studies.
\end{abstract}

Ke y words: Staphylococcus hominis, epidemiological studies, BOX-PCR fingerprinting, MLVA analysis

Coagulase-negative Staphylococcus hominis is a part of the commensal bacterial microflora of healthy people. Simultaneously, these bacteria are recognized as important causes of nosocomial infections, especially in patients with indwelling prosthetic devices and in immunocompromised neonates (Chaves et al., 2005; Götz 2006; Kaufman and Fairchild, 2004; Rodhe etal., 2006). This bacterium can be responsible for bloodstream infections, sepsis, endocarditis, peritonitis, bone and joint infections (Kloos and Bannerman, 1999). Similar to other staphylococci, the formation of stable biofilm on medical devices or on host tissues is thought to be the major pathogenic factor of S. hominis (Chokr et al., 2006; Fredheim et al., 2009). Many studies revealed that distinct staphylococci clones can become endemic and that these clones can persist in the hospital environment for many years (Kelly et al., 2008; Muldrew et al., 2008; Rodríguez-Aranda et al., 2009; Treviño et al., 2009). On the other hand, it has been observed that staphylococcal isolates obtained in hospitals display a wide genetic diversity (Fredheim et al., 2009; Nunes et al., 2005; Szczuka et al., 2010, Szczuka et al., 2012). This could be due to the fact that the pathogens do not represent a single evolutionary line but rather they are composed of several lines (Melless et al., 2004; Miragaia et al., 2008; Rijnders et al., 2009; Pupo et al., 1997; Szczuka and Kaznowski 2004). Although S. hominis is the third most commonly isolated species among CoNS, there is limited information available on the epidemiology of S. hominis. There is also a lack of easy-to-use and rapid methods for molecular typing of S. hominis. Pulsed-field gel electrophoresis (PFGE) is considered the "gold standard" for molecular typing of coagulase-negative staphylococci. However, this method has the drawback of being time-consuming, subjective in interpretation, technically demanding and relatively expensive (Miragaia et al., 2008; Wang et al., 2003). Multilocus sequence typing (MLST) measures genetic change for housekeeping genes, which evolve extremely slowly and for this reason this method is suitable for long-term global epidemiology of Staphylococcus epidermidis (Wang et al., 2003). In view of the above, alternative methods have been proposed. Repetitive-element PCR (rep-PCR) analysis based on multicopy elements of the staphylococcal genome has good reproducibility and discriminatory power (Krzymińska et al., 2012) and recently rep-PCR has been commercially adapted to automated format known as the DiversiLab System (bioMérieux, France) (Treviño et al., 2009). Recently, Johansson et al. (2006) proposed multiple-locus variable-number tandem repeat analysis (MLVA) for the discrimination of clinical isolates of

* Corresponding author: E. Szczuka, Department of Microbiology, Institute of Experimental Biology, Faculty of Biology, Adam Mickiewicz University, ul. Umultowska 89, 61-614 Poznań, Poland; phone: +48 (61) 8295937; fax: +48 (61) 8295550; e-mail: ewasz@amu.edu.pl 
S. epidermidis. This method relies on the detection of different copy numbers of short repeated sequence that are arranged in tandem arrays.

The purpose of this study was to determine the genetic relationship among $S$. hominis strains isolated from clinical specimens from patients treated in a hospital in 2001-2012. Clones have been differentiated by repetitive sequence based PCR method (BOX-PCR) and multiple-locus variable-number tandem repeat analysis (MLVA).

Clinical isolates of S.hominis were obtained in the period 2001-2012 from clinical specimens from patients treated in different departments of a 700-bed Regional Hospital in Poznań, Poland. Strains were recovered from well-defined infections in patients. Identification of bacterial cultures was performed using the Vitek 2 system (bioMérieux, France). The reference strain of S. hominis ATCC 2122 was used in this study.

Bacteria were grown on blood agar at $37^{\circ} \mathrm{C}$ overnight, and DNA was extracted with the Genomic DNA Plus kit (A\&A Biotechnology, Poland). The PCR reaction was performed using primer with the sequence (5'CTACGGCAAGGCGACGCTGACG3') complementary to BOX elements of bacterial genomic DNA (Versalovic et al., 1991). The PCR was performed by methods described by Wieser and Busse (2000). The resulting fragments were electrophoresed in $1.5 \%$ agarose gel. The DNA in gels was stained with ethidium bromide, visualized on a UV light transilluminator, and documented with V.99 Bio-Print system (Vilber Lourmat, Torcy, France).

The sequence of S. hominis SK 119 was downloaded from the National Center for Biotechnology Information (NCBI) and analyzed for the presence of tandem repeat sequences with the software program Tandem Repeats Finder. The analysis identified 142 genomic regions with tandem repeat sequences and eleven regions were selected for further analysis on the basis of genomic location, repeat length, and copy number. These tandem repeat sequences showed a minimum size of $18 \mathrm{bp}$ for an individual repeat and a tandem copy number greater than two. Flanking primers were designed for the repeat loci based on the genome sequence of the SK 119 S. hominis strain. Using the Basic Local Alignment Search Tool (BLAST) we searched for the repeated sequence in other C80 S. hominis strain. The three repeated sequences were not found and for this reason these sequences were excluded from the studies. PCR was performed by the method described by Johansson et al. (2006). When performing MLVA, we noted PCR amplification failure at six loci (desig- nated as Sh 3, Sh 4, Sh 5, Sh 7, Sh 8 and Sh 11) for many $S$. hominis isolates. The easiest explanation for this is that the targeted sequence is missing in these strains. PCR amplification at loci Sh 1 and Sh 2 were successful. The multiplex PCR was performed for the repeat motif TTTTGATTTGATTTTGATTT (locus Sh 1) and repeat motif TTCTTTAGATTTCTTATC (locus Sh 2) using primers F-, GTTGCTTTGGCGTTTTGTTT and R-TTGAGGAGGCACCAATAACA for locus Sh 1 and F-TGCTGCTCTTGAGTTGCAGT and R-GCTTGTAGTCAAGATGACGCTAAA for locus Sh 2.

The results of the BOX and MLVA were analyzed using GelCompar II (version 3.0; Applied Maths, Kortrijk, Belgium) software. The percentages of fingerprint similarities were analyzed using the Dice coefficient. The UPGMA clustering method was used to create a dendrogram. The results of the MLVA and BOX analysis are presented in Fig. 1. and Fig. 2.

On the basis of BOX-PCR, sixty three genotypes were identified. The fingerprints of the isolates consisted of 8 to 14 bands, indicating the presence of several copies of the BOX sequence in the genomes of $S$. hominis strains. All strains showed a high degree of genetic diversity and had unique BOX-PCR patterns. The patterns of all S. hominis strains showed very low similarity $(S=26 \%)$. MLVA analysis allocated thirty nine isolates to 10 clusters, when a similarity level of $95 \%$ was applied. Strains grouped in these clusters were isolated from people treated in different wards of the hospital. We did not observe distribution of these strains into the clusters according to their source of isolation. Twenty four isolates showed unique MLVA patterns. The largest cluster was identified on the basis of one DNA fragment. Other clusters were designed on the basis of two or three DNA fragments. The results indicated that there was no sufficient variation in repeated regions to make them suitable candidates for sequence-based typing. It appears that strains showing the same MLVA patterns had completely different BOX banding patterns. Thus, we can conclude that MLVA is not as discriminatory as BOX analysis and can not be recommended as an epidemiological tool.

Despite the growing importance of S. hominis as a cause of nosocomial infections, there is limited information available on the epidemiology of S. hominis in the hospital setting. Our results revealed a large genetic diversity of S. hominis strains obtained from patients treated in one hospital over 11 years. We did not identify an endemic clone of S.hominis in the hospital setting with the ability to spread epidemically. Thus, patients were infected with clonally-unrelated strains

Fig. 1. Dendrogram generated from BOX-PCR profiles of S. hominis strains by the UPGMA with arithmetic means. The Dice band-based similarity coefficient was calculated with a band position tolerance of $1 \%$ 
BOX - PCR

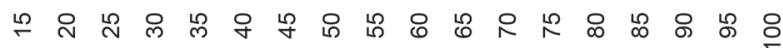

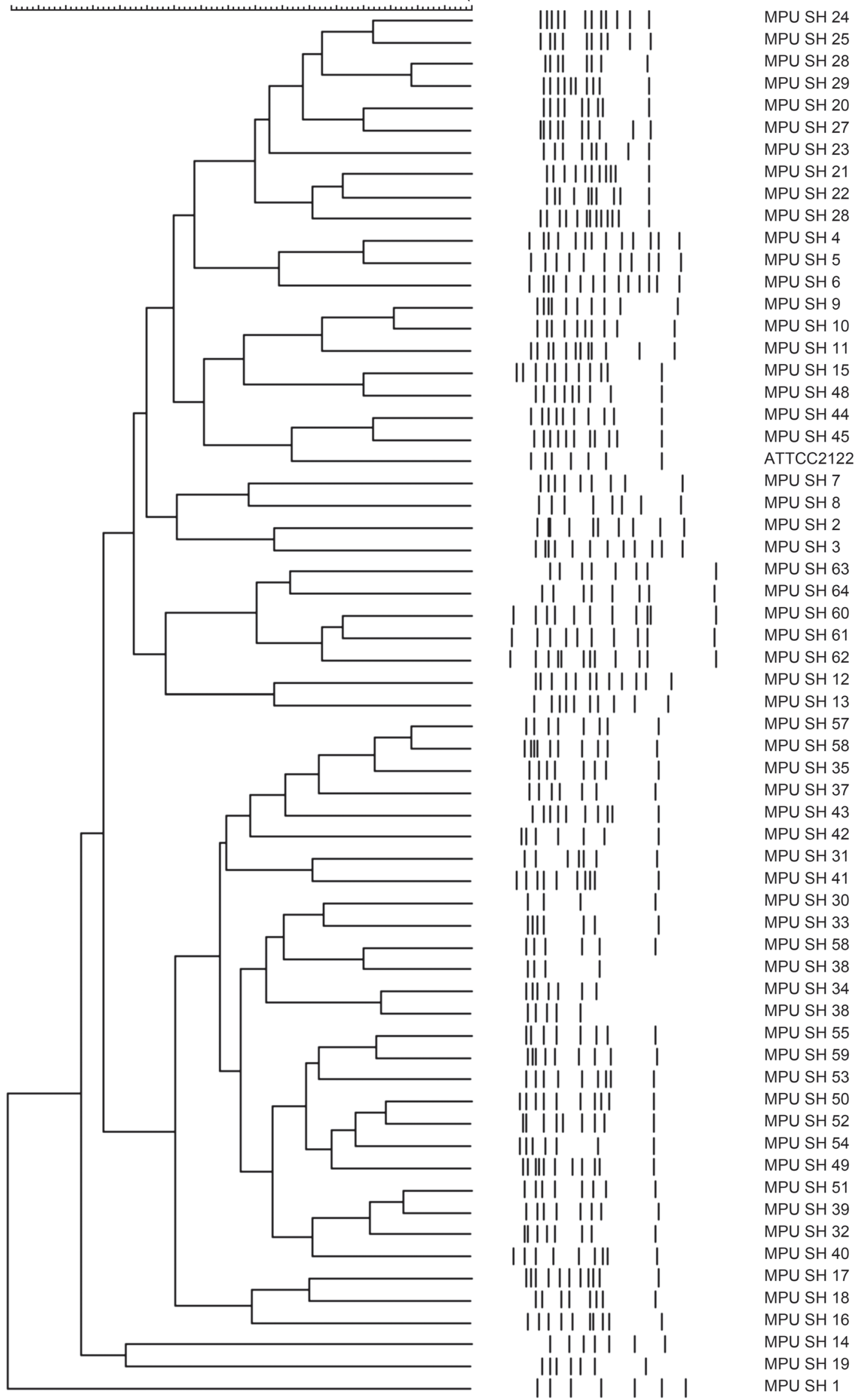




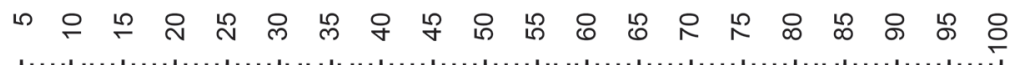

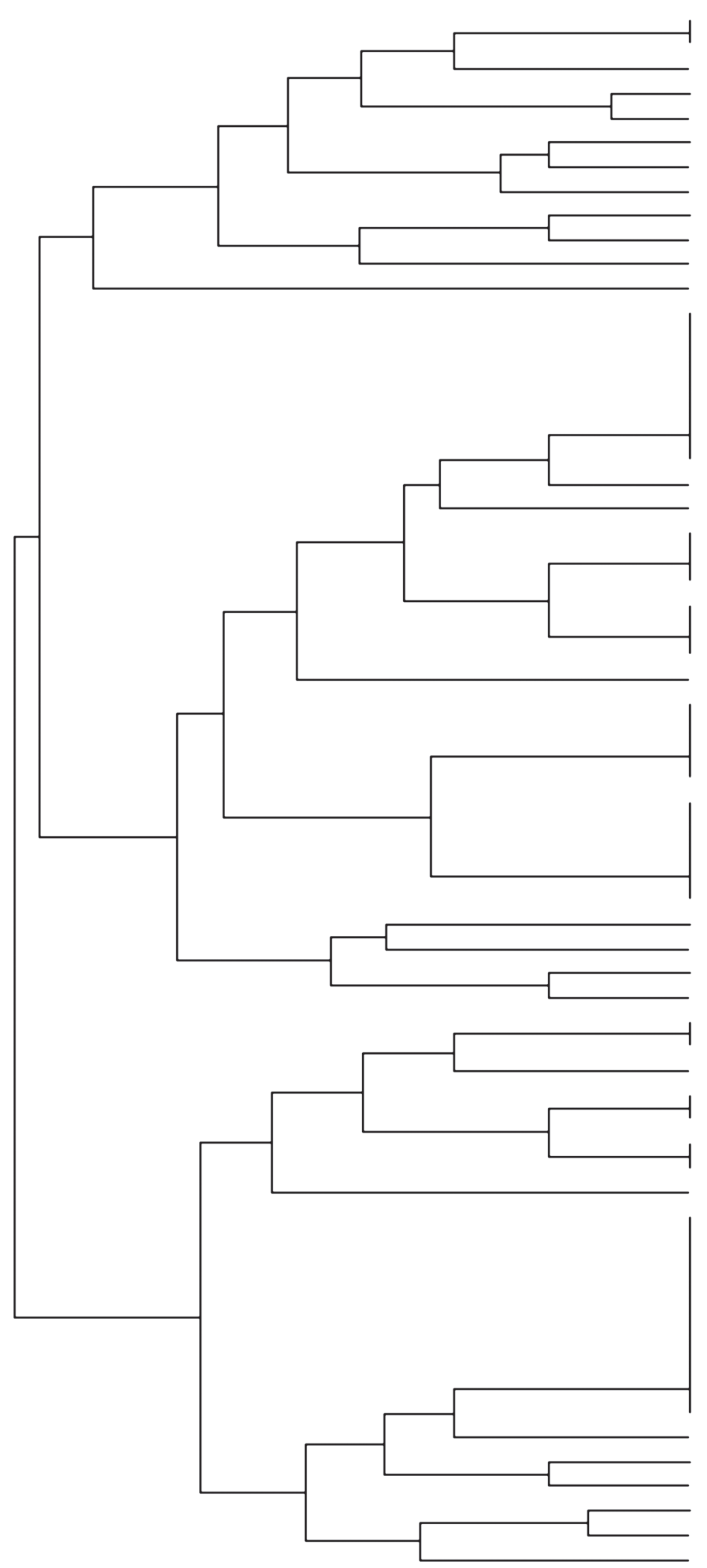

MLVA

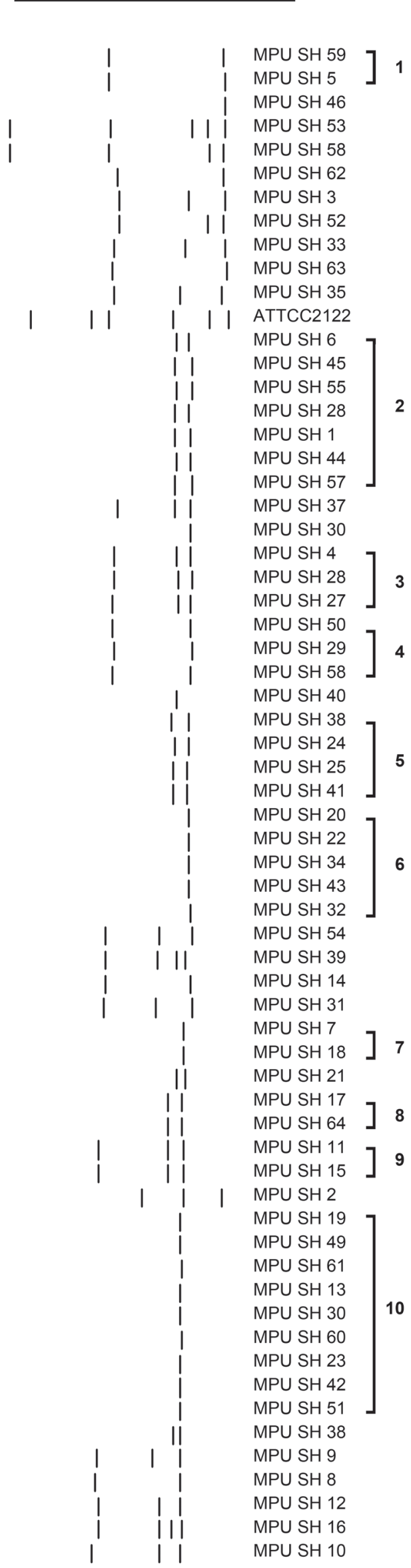


and none of these strains persisted in the hospital environment. Previously, Chaieb etal. (2006) found that a single clone of S. hominis has been responsible for sepsis of thirteen neonates treated at a neonatology department in a Madrid hospital. In other reports, only a few $S$. hominis were investigated and these strains were grouped with other CoNS species using PFGE (de Silva et al., 2002; Chaieb etal., 2006; Spare et al., 2003). In our opinion, an accurate, fast and reproducible typing system would improve the epidemiological investigation of S. hominis. BOX-PCR appeared to be the more suitable method for epidemiological studies than MLVA. Strain differentiation by means of MLVA is very often based on single amplicon or two amplicons in the MLVA profiles. The result of this study demonstrated that the repeated regions chosen for MLVA analysis did not display sufficient variation for the discrimination of $S$. hominis isolates. BOX profiles as well as MVLA profiles did not contain a species-specific band and therefore these analyses cannot be used for identification of the $S$. hominis strains.

In conclusion, we observed a wide genetic diversity among S.hominis strains isolated from hospitalised patients treated in one hospital. BOX typing system is efficient in the analysis of large numbers of isolates and therefore suitable for epidemiological studies as well as for the detections of relatedness of isolates.

\section{Acknowledgements}

The authors would like to express their gratitude to the staff of Department of Microbiological and Laboratory Diagnostics, Bacteriological Laboratory, Regional Hospital in Poznań, for the isolates of $S$. hominis they have so kindly provided.

\section{Literature}

Chaieb K., A. Touati, A.M. Salah, A.B. Hassen, K. Mahdouani and A. Bakhrouf. 2006. DNA fingerprinting of a multi-resistant coagulase-negative staphylococci isolated from biomaterials in dialysis services. Arch. Med. Res. 37: 953-960.

Chaves F., M. García-Álvarez, F. Sanz, C. Alba and J.R. Otero. 2005. Nosocomial spread of a Staphylococcus hominis subsp. novobiosepticus strain causing sepsis in a neonatal intensive care unit. J. Clin. Microbiol. 43: 4877-4879.

Chokr A., D. Watier, H. Eleaume, B. Pangon, J.C. Ghnassia, D. Mack and S. Jabbouri. 2006. Correlation between biofilm formation and production of polysaccharide intercellular adhesin in clinical isolates of coagulase-negative staphylococci. Int. J. Med. Microbiol. 296: 381-388.

de Silva GD., M. Kantzanou, A. Justice, R.C. Massey, A.R. Wilkinson, N.P. Day and S.J. Peacock. 2002. The ica operon and biofilm production in coagulase-negative Staphylococci associated with carriage and disease in a neonatal intensive care unit. J. Clin. Microbiol. 40: $382-388$.
Götz F. 2006. The genera Staphylococcus and Macrococcus, pp. 5-75. In: Dworkin M., S. Falkow, E. Rosenberig, K-H. Schleiferr, E. Stackebrands (eds). The Prokaryotes, $3^{\text {nd }}$ A Handbook on the biology of bacteria: Firmicutes, Cyanobacteria.

Fredheim E.G.A., C. Klingenberg, H. Rodhe, S. Frankenberger, P. Gaustad, T. Fllaegstad and E. Sollid. 2009. Biofilm formation by Staphylococcus haemolyticus. J. Clin. Microbiol. 47: 1172-1180.

Johansson A., S. Koskiniemi, P. Gottfridsson, J. Wiström and T. Monsun. 2006. Multiple-Locus Variable-Number Tandem Repeat Analysis for typing of Staphylococcus epidermidis. J. Clin. Microbiol. 44: 260-265.

Kaufman D. and D. Fairchild. 2004. Clinical microbiology of bacterial and fugal sepsis in very-low-birth-weight infants. Clin. Microbiol. Rev. 17: 638-680.

Kelly S., J. Collins, M. Maguire, C. Gowing, M. Flanagan, M. Donnelly and P.G. Murphy. 2008. An outbreak of colonization with linezolid-resistant Staphylococcus epidermidis in an intensive therapy unit. J. Antimicrob. Chemother. 61: 901-907.

Kloos W.E. and T.L. Bannerman. 1999. Staphylococcus and Micrococcus. pp 264-282. In: Murray P.R., E. Baron, M.A. Pfallen, F.C. Tenover and R. Yolken, R. (eds) Manual of Clinical Microbiology. ASM Press, Washington, D.C.

Krzymińska S., E. Szczuka and A. Kaznowski. 2012. Staphylococcus haemolyticus strains target mitochondria and induce caspasedependent apoptosis of macrophages. Antonie van Leeuwenhoek 102: 611-620.

Melles D.C., R.F. Gorkink, H.A. Boelens, S.V. Snijders, J.K. Peeters, M.J. Moorhouse, P.J. van der Spek, W.B. van Leeuwen, G. Simons, H.A. Verbrugh and A.van Belkum. 2004. Natural population dynamics and expansion of pathogenic clones of Staphylococcus aureus. J. Clin. Invest. 114: 1732-1740.

Miragaia M, J.A. Carric, J.C. Thomas, I. Couto, M.C. Enright and H. de Lencastre. 2008. Comparison of Molecular Typing Methods for Characterization of Staphylococcus epidermidis: Proposal for Clone Definition. J. Clin. Microbiol. 46: 118-129.

Muldrew K.L., Y-W. Tang, H. Li and C.W. Stratton. 2008. Clonal dissemination of Staphylococcus epidermidis in an oncology ward. J. Clin. Microbiol. 46: 3391-3396.

Nunes A.P., L.M. Teixeira, C.C. Bastos, M.G. Silva, R.B. Ferreira, L.S. Fonseca and K.R. Santos. 2005. Genomic characterization of oxacillin-resistant Staphylococcus epidermidis and Staphylococcus haemolyticus isolated from Brazilian medical centres. J Hosp. Infect. 59: 19-26.

Pupo, G.M., D.K. Karaolis, R. Lan, and P.R. Reeves. 1997. Evolutionary relationships among pathogenic and nonpathogenic Escherichia coli strains inferred from multilocus enzyme electrophoresis and mdh sequence studies. Infect. Immun. 65: 2685-2692.

Rijnders M.I.A., R.H. Deurenberg, M.L.L. Boumans, J.A.A. Hoogkamp-Korstanje, P.S. Beisser, the Antibiotic Resistance Surveillance Group and E. E. Stobberingh. 2009. Population structure of Staphylococcus aureus strains isolated from intensive care unit patients in The Netherlands over an 11-year period (1996 to 2006). J. Clin. Microbiol. 47: 4090-4095.

Rodhe H., D. Mack, M. Christner, C. Burdelski, G. Franke and J.K-M. Knobloch. 2006. Pathogenesis of staphylococcal devicerelated infections: from basic science to new diagnostic, therapeutic and prophylactic approaches. Rev. Med. Microbiol. 17: 45-54.

Rodríguez-Aranda A., M. Daskalaki, J. Villar, F. Sanz, J.R. Otero and F. Chaves. 2009. Nosocomial spread of linezolid-resistant Staphylococcus haemolyticus infections in an intensive care unit. Diagn. Microbiol. Infect. Dis. 63: 398-402.

Fig. 2. Dendrogram generated from MLVA profiles of S. hominis strains by the UPGMA with arithmetic means. The Dice band-based similarity coefficient was calculated with a band position tolerance of $1 \%$ 
Spare M.K., S.E. Tebbs, S. Lang, P.A. Lambert, T. Worthington, G.W. Lipkin and T.S. Elliott. 2003. Genotypic and phenotypic properties of coagulase-negative staphylococci causing dialysis catheter-related sepsis. J. Hosp. Infect. 54: 272-278.

Szczuka E. and A. Kaznowski. 2004. Typing of clinical and environmental Aeromonas sp. strains by Random Amplified Polymorphic DNA PCR, Repetitive Extragenic Palindromic PCR, and Enterobacterial Repetitive Intergenic Consensus Sequence PCR. J. Clin. Microbiol. 42: 220-228.

Szczuka E., A. Szumała-Kąkol, A. Kaznowski and A. Siuda. 2010. Clonal analysis of methicillin-sensitive Staphylococcus aureus strains isolated in obstetric-gynaecological hospital. Pol. J. Microbiol. 59: 161-165.

Szczuka E., K. Urbańska, M. Pietryka and A. Kaznowski. 2012. Biofilm density and detection of biofilm-producing genes in methi- cillin-resistant Staphylococcus aureus strains. Folia Microbiol DOI 10.1007/s12223-012-0175-9

Treviño M., L. Martínez-Lamas, P.A. Romero-Jung, J.M.Giráldez, J. Alvarez-Escudero, and B.J. Regueiro. 2009. Endemic linezolidresistant Staphylococcus epidermidis in a critical care unit. Eur. J. Clin. Microbiol. Infect. Dis. 28: 527-533.

Versalovic J., T. Koeuth and J.R. Lupski. 1991. Distribution of repetitive DNA sequences in eubacteria and application to fingerprinting of bacterial genomes. Nucleic Acids Res. 19: 6823-6831.

Wang X.M., L. Noble, B.N. Kreiswirth, W. Eisner, W. McClements, K.U. Jansen and A.S. Anderson. 2003. Evaluation of a multilocus sequence typing system for Staphylococcus epidermidis. J. Med. Microbiol. 52: 989-998.

Wieser M. and H-J. Busse. 2000. Rapid identification of Staphylococcus epidermidis. 2000. Int. J. Syst. Evol. Microbiol. 50: 1087-1093. 